\title{
Prevention of catheter-associated urinary tract infection
}

\author{
Barbara W. Trautnera, ${ }^{a, b}$ Richard A. Hull ${ }^{b, c}$, and Rabih O. Darouiche ${ }^{a, b}$ \\ a Department of Medicine, Infectious Diseases Section, Michael E. DeBakey Veterans Affairs \\ Medical Center and Baylor College of Medicine, Houston, Texas, USA \\ b Department of Physical Medicine and Rehabilitation, Center for Prostheses Infection, Michael E. \\ DeBakey Veterans Affairs Medical Center and Baylor College of Medicine, Houston, Texas, USA \\ c Department of Molecular Virology and Microbiology, Baylor College of Medicine, Houston, \\ Texas, USA
}

\begin{abstract}
Purpose of review-The underlying cause of catheter-associated urinary tract infection is biofilm formation by uropathogens on the urinary catheter. Biofilm is a relatively new concept in medicine, and current measures to prevent biofilm formation are inadequate. Considerable work is being done in this area, but little clinical progress has been made. The purpose of this review is to analyze recent publications concerning prevention of catheter-associated urinary tract infection.

Recent findings-Several recent studies have elucidated aspects of biofilm formation in catheter-associated urinary tract infection. Other researchers are working on methods to disrupt biofilm formation on catheter surfaces. At the same time, the magnitude of the problem of catheter-associated urinary tract infection has increased awareness of the effectiveness of basic infection control measures. A modern approach to infection control may include computerized ordering systems that minimize unnecessary days of catheterization. Finally, consumption of cranberry juice products and bacterial interference are two novel approaches to urinary tract infection prevention.
\end{abstract}

Summary-Biofilm-disrupting strategies offer promise for the future but have little immediate applicability. Implementation of infection control measures to improve catheter function and remove unnecessary catheters can be done at the present time. In general, prevention of catheterassociated urinary tract infection remains an elusive goal. More basic research at the level of pathogenesis is needed so that novel strategies can be designed.

\section{Keywords}

biofilm; urinary catheter; urinary tract infection

\section{Introduction}

Catheter-associated urinary tract infection (CAUTI) is a common problem with considerable economic impact $[1,2 \bullet]$. The underlying cause of CAUTI is formation of a pathogenic biofilm on the surface of the indwelling urinary catheter, and currently we have few, if any, effective strategies to impede this process. 
Understanding the pathogenesis of CAUTI is essential to designing and evaluating preventative strategies. A foreign body in a moist environment, such as a urinary catheter in the bladder, inevitably becomes colonized with sessile microorganisms living in a biofilm community [3]. Thus, individuals with an indwelling urinary catheter develop bacteriuria at the rate of 3-10\% per day [4], and the incidence of bacteriuria approaches $100 \%$ in individuals who are chronically catheterized [5]. The biofilm lifestyle conveys a survival advantage to micro-organisms in terms of their ability to withstand drying, shear forces, ultraviolet radiation, and antimicrobial agents. The practical consequence is that an indwelling urinary catheter cannot usually be cleared of a pathogenic biofilm without removing the catheter. As long as the colonized catheter remains in place, biofilm-associated organisms can seed the urine with bacteria.

A clear understanding of the distinction between urinary tract infection (UTI) and asymptomatic bacteriuria is essential to the critical assessment of the literature concerning prevention of CAUTI. The presence of bacteria in the bladder, a normally sterile site, triggers an inflammatory response [6-8]. However, more than $90 \%$ of cases of nosocomial catheter-associated bacteriuria are asymptomatic, or subclinical infections $[1,9 \bullet]$. The presence of bacteria in the urine of an asymptomatic person is termed asymptomatic bacteriuria and is distinguished from a symptomatic UTI by the absence of symptoms attributable to infection of the urinary tract. Since the majority of cases of asymptomatic bacteriuria do not progress to UTI, clinical trials that use bacteriuria as an endpoint are methodologically flawed. Another common misunderstanding is that measures to prevent bacteriuria during short-term catheterization ( $\leq 14$ days) are erroneously assumed to be effective at preventing UTI in persons with chronic, indwelling urinary catheters ( $>30$ days).

The purpose of this article is to analyze the reports published in the past year on prevention of CAUTI. Several studies have elucidated aspects of biofilm formation; these studies advance the field by contributing to a better understanding of pathogenesis. Other basic science studies have explored techniques to disrupt biofilm formation on medical devices in general. Although these ideas show promise, the only biofilm-impeding strategy that has been tested in clinical trials is impregnating urinary catheters with antimicrobial silver. Recent publications also reiterate that attention to infection control measures helps prevent CAUTI. Finally, two novel approaches to UTI prevention in general have recently reached the stage of clinical trial: consumption of cranberry products and bacterial interference.

\section{Understanding the pathogenesis of catheter-associated urinary tract infection}

Several publications from the past year elucidate the pathogenesis of CAUTI. Sabbuba and colleagues [10•] used molecular epidemiology to prospectively study Proteus mirabilis in the urinary flora of catheterized patients. A striking finding was that the same strain of Proteus colonized one patient for 121 days despite eight catheter changes, an 8-day course of antibiotics (which temporarily eliminated $P$. mirabilis from the urine), and a 20-day period during which the bladder was not catheterized. This finding suggests that uropathogens may persist in a reservoir within the urinary tract itself, rather than re-entering from the stool; the latter mechanism would be expected to result in bladder invasion by different strains.

A recent publication in Science [11••] suggests that this reservoir may be within the living tissue of the bladder itself. Anderson et al. found that clinical isolates of uropathogenic Escherichia coli formed tightly-packed, biofilm-like pods in mouse bladder epithelial cells. This article is the first description of biofilm formation within eukaryotic cells, and the accompanying images are striking. If supported by further studies, these intracellular 
biofilms could certainly account for the persistence of pathogens in the damaged mucosa of a catheterized urinary tract [12]. A refined understanding of the pathogenesis of CAUTI may lead to novel mechanisms to prevent this disease.

\section{Disrupting biofilm formation with novel catheter coatings}

Since biofilm on the urinary catheter is the central factor in pathogenesis of CAUTI, many scientists seek to alter the catheter surface in order to inhibit biofilm formation. No surface can resist biofilm formation indefinitely in the urinary tract, but impeding biofilm formation may suffice if the catheter is intended for short term use. Readers seeking background information about biomaterials currently licensed for use in the urinary tract should refer to a review article by Beiko et al. in the Journal of Urology [13•].

The type of urinary catheter modification that has undergone the most extensive clinical testing is impregnating the catheter with antimicrobial agents, most commonly with silver. In 2004 the Cochrane Database of Systemic Reviews published a comprehensive assessment of impregnated catheters intended for short-term use in hospitalized adults [14••]. Eight differently-designed trials compared silver alloy catheters with standard catheters. Pooled results indicated that the risk of asymptomatic bacteriuria was significantly reduced in the silver alloy group at less than 1 week of catheterization (RR 0.36 ; 95\% CI $0.25-0.52$ ) and, to a lesser degree, at greater than 1 week (RR $0.67 ; 95 \%$ CI 0.50-0.90). The risk of symptomatic UTI was also lower in the groups with silver alloy catheters (RR 0.60; 95\% CI $0.50-0.73$ ), although the symptoms used to define UTI were not specified. None of the trials tested for emergence of resistance to silver. The Cochrane reviewers noted that methods of randomization and blinding were unclear, various types of silver alloy coatings were used, and only one trial did a cost-benefit analysis [15].

What are we to conclude about the use of antimicrobial-impregnated urinary catheters? Silver alloy catheters temporarily delay the onset of bacteriuria, as evinced by the increase in bacteriuria between 1 week of catheterization and greater than 1 week of catheterization. Systemic antimicrobial prophylaxis can have the same effect. However, systemic antimicrobial agents are not used to prevent nosocomial CAUTI because the benefits of preventing largely asymptomatic bacteriuria are dubious and the risk of selecting resistant flora is high [16]. Silver-resistant bacteria are common in environments where silver antiseptics are widely used, such as in burn units [17•]. To summarize, the use of the more expensive, silver-coated catheters to prevent CAUTI is not supported by quality data, and resistance to silver is likely to become a problem with widespread use. These concerns are applicable to other agents used to impregnate urinary catheters, including chlorhexidine [18•] and nitrofuroxone [19•]. Currently the guiding principle in selecting a urinary catheter should be patient comfort; both hydrophilic coatings and all-silicone surfaces reduce frictional irritation of the urethra $[13 \bullet, 20 \bullet]$.

Many substances are being tested for their potential as biofilm-disrupting catheter coatings. Some of these substances have been recently discovered, such as quorum-sensing inhibitors, while others are currently used for different medical purposes. The most recent studies of the use of the quorum-sensing inhibitors to prevent biofilm formation have been done with staphylococcal graft infections $[21 \bullet, 22 \bullet$. Coating dacron vascular catheters with RNAIIIinhibiting peptide, a peptide that disrupts staphylococcal cell-cell communication, reduced graft infection in a rat model [23•]. Although this work was done with vascular catheters, the concept is relevant to urinary catheter infections; quorum-sensing regulation of biofilm formation is well-described in Pseudomonas [24] and Escherichia coli [25], both common agents of CAUTI. None of the quorum-sensing inhibitors have been tested in humans. 
Several agents with a long history of medical use in humans are currently being investigated for the purpose of controlling urinary catheter-associated biofilms. These agents include heparin, $\mathrm{N}$-acetylcysteine, and aspirin. An in-vitro study of urinary catheters exposed to Proteus mirabilis showed that the time to encrustation was significantly longer for heparincoated catheters than for control hydrogel-coated latex catheters $(28.1 \mathrm{~h}$ versus $58.2 \mathrm{~h}$, respectively) [26•]. These same authors performed a pilot study in 20 patients and found that heparin-coated stents developed less encrustation over 2-6 weeks than control stents [26•]. Two other studies added a potential anti-biofilm agent to the microbial growth medium rather than to the catheter surface. In one study [27•] N-acetylcysteine, a mucolytic agent, reduced biofilm formation by $E$. coli on polystyrene wells, and in the other study [28•] aspirin inhibited biofilm formation by Candida albicans on polyvinyl chloride. A weakness of the latter two trials is that the effect of the agent on biofilm formation was not clearly distinguished from the effect of the agent on viability of the pathogens. Although these established agents may eventually prove to be anti-biofilm drugs, at this time no clinical data exists to support their use for prevention of CAUTI.

\section{Infection control measures}

Currently one well-tested strategy is available that can prevent catheter-associated infections. This often neglected strategy is to implement infection control measures to ensure that urinary catheters are maintained properly and removed promptly when no longer needed.

Ensuring unobstructed flow of urine is one of the most effective measures to prevent CAUTI. A recent study [29•] in persons with spinal cord injury using intermittent catheterization for bladder drainage confirmed that the risk of UTI increases with the amount of residual urine in the bladder. Similarly, an investigation performed by a large home-nursing care agency found that catheter blockage and low urine flow were significantly associated with CAUTI [30•]. The concept that catheter obstruction leads to stagnant urine, an overdistended bladder, and UTI is hardly novel, yet how many hospitals have a mechanism to ensure gravity-dependent, unobstructed drainage at all times? A study in two intensive care units in a hospital in Buenos Aires, Argentina [31•] tested the effect of implementing two infection control practices: (1) handwashing before insertion of a urinary catheter and (2) positioning the catheter so that it was not compressed by the patient's leg. During the 21-month intervention period, compliance with both infection control measures increased significantly, and the rate of CAUTI decreased significantly from 21.3 to 12.4 per 1000 catheter-days (RR, $0.58 ; 95 \%$ CI $0.39-0.86 ; P=0.006)$. Likewise, in an intensive care unit in Paris, implementation of an infection control program that ensured unobstructed catheter flow led to a decrease in CAUTI from 21.1 to 12.9 per 1000 catheter days over the 5 years of the study $(P=0.0006)[32 \cdot]$. While neither of these studies proves that the decreased incidence of UTI over the trial period was due solely to changes in the infection control practices, the results of both trials argue strongly for attention to these simple, affordable measures.

Another effective means to prevent CAUTI is to avoid unnecessary days of catheterization. Approximately $30 \%$ of initial urinary catheterizations are unjustified, and one-third to onehalf of days of continued catheterization are unjustified [33-35]. Many of these catheters are inserted in the emergency room without a documented order, and providers are not aware that the catheter is in place in $21-38 \%$ of cases [35]. A logical solution to this problem would be to require entry of a computer order for each urinary catheter. The computer system could request the indication for catheterization and prompt an automatic catheter removal date. A recent controlled study of such an ordering system was found to decrease 
the duration of catheterization by 3 days (from 8 to 5 days' $P=0.03$ ) [36••]. This promising strategy awaits testing in a full-scale trial designed to measure the impact on CAUTI.

\section{Novel methods to prevent urinary tract infection in general}

Two novel methods to prevent urinary tract infection, not specifically CAUTI, have been tested in clinical trials in humans: consumption of cranberry products and bacterial interference (using nonpathogenic bacteria to prevent infection by pathogens).

The Cochrane Database of Systemic Reviews recently published an analysis of cranberry products for prevention of UTI [37••]. Overall, the amount and type of cranberry products given to participants varied widely. Of seven trials that met inclusion criteria, only two randomized, controlled studies were suitable for meta-analysis [38,39]. The summary risk ratio for cranberry products (juice or capsules) versus placebo for recurrent UTI in the 300 adult, non-catheterized women in these two trials was 0.61 (95\% CI 0.40-0.91). The high dropout rates suggested that cranberry juice consumption was not a well-accepted therapy [40•]. Thus, the clinical evidence supporting cranberry juice for prevention of UTI is weak.

Bacterial interference is unique among preventative approaches in that the goal of bacterial interference is to select benign organisms to form a catheter-associated biofilm rather than to prevent biofilm formation. The appeal of this concept is that humans do not have to outsmart the bacteria, who throughout history have repeatedly demonstrated their ability to evade human defenses. A pilot trial of direct instillation of a benign strain of E. coli into the neurogenic bladders of persons with spinal cord injury showed that direct bladder instillation was safe, did not produce symptoms of UTI, and reduced the frequency of UTI as compared with the patients' historical baselines [41]. A multicenter, prospective, placebo-controlled trial of direct bladder instillation of benign E. coli to prevent UTI in persons with spinal cord injury began recently. In vitro, incubating urinary catheters with this same strain of nonpathogenic E. coli prior to exposing the catheters to a variety of uropathogens effectively impeded catheter colonization by uropathogens $[42,43 \bullet]$. A pilot trial of inserting E. colicoated urinary catheters into the bladders of persons with spinal cord injury is underway. Since biofilms on urinary catheters are notoriously difficult to eradicate, perhaps we can exploit biofilm formation in order to induce durable bladder colonization with benign flora.

\section{Conclusion}

CAUTI is a significant problem for the millions of persons who receive urinary catheters each year. Unfortunately, the most effective preventative strategy developed to date is the use of a closed drainage system, introduced almost a century ago [44]. The next most effective strategy is to remove the catheter as soon as it is no longer needed, a maneuver which may be prompted by automated computer stop orders [36・0]. In addition to these two measures, strict attention to infection control practices, especially ensuring gravitydependent drainage of urine, can also decrease CAUTI. All of the forgoing measures require little expenditure and can be implemented immediately.

Techniques for preventing formation of a pathogenic biofilm on the urinary catheter surface are being developed; none has proven clinical efficacy. Bacterial interference is an antimicrobial-sparing approach that may prevent CAUTI by colonizing the urinary catheter and the bladder with benign flora. Basic science research continues to elucidate the complexity of biofilm formation in the catheterized urinary tract, thereby encouraging the development of novel means to prevent CAUTI. 


\section{Acknowledgments}

This study was supported by the Department of Veterans Affairs Rehabilitation Research and Development Service Merit Review \#B2125-RA, Paralyzed Veterans of America Grant 302, and USPHS Grant HD42014.

\section{Abbreviations \\ CAUTI catheter-associated urinary tract infection \\ UTI urinary tract infection}

\section{References and recommended reading}

Papers of particular interest, published within the annual period of review, have been highlighted as:

- of special interest

$\bullet$ of outstanding interest

1. Tambyah PA, Maki DG. Catheter-associated urinary tract infection is rarely symptomatic. Arch Intern Med. 2000; 160:678-682. [PubMed: 10724054]

2-. Saint S, Chenoweth CE. Biofilms and catheter-associated urinary tract infections. Infect Dis Clin North Am. 2003; 17:411-432. [PubMed: 12848477] This paper is an excellent review of the role of biofilm in CAUTI and also addresses the risk of UTI associated with various urine collection strategies (indwelling, condom, and suprapubic catheters).

3. Denstedt JD, Wollin TA, Reid G. Biomaterials used in urology: current issues of biocompatibility, infection, and encrustation. J Endourol. 1998; 12:493-500. [PubMed: 9895250]

4. Saint S, Lipsky BA, Goold SD. Indwelling urinary catheters: a one-point restraint? Ann Intern Med. 2002; 137:125-128. [PubMed: 12118969]

5. Warren JW, Tenney JH, Hoopes JM, et al. A prospective microbiologic study of bacteriuria in patients with chronic indwelling urethral catheters. J Infect Dis. 1982; 146:719-723. [PubMed: 6815281]

6. Musher DM, Thorsteinsson SB, Airola VM. Quantitative urinalysis: diagnosing urinary tract infection in men. JAMA. 1976; 236:2069-2072. [PubMed: 989790]

7. Agace WW, Hedges SR, Ceska M, Svanborg C. Interleukin-8 and the neutrophil response to mucosal gram-negative infection. J Clin Invest. 1993; 92:780. [PubMed: 8349817]

8. Hedges S, Anderson P, Lidin-Janson G, et al. Interleukin-6 response to deliberate colonization of the human urinary tract with gram-negative bacteria. Infect Immun. 1991; 59:421. [PubMed: 1987054]

9•. Daniel, M Musher. How contagious are common respiratory tract infections? N Engl J Med. 2003; 348:1256-1266. [PubMed: 12660390] This article concerns respiratory infections, but it neatly addresses the distinction between infection and colonization in its introduction.

10•. Sabbuba NA, Mahenthiralingam E, Stickler DJ. Molecular epidemiology of Proteus mirabilis infections of the catheterized urinary tract. J Clin Microbiol. 2003; 41:4961-4965. [PubMed: 14605124] These researchers used pulsed-field gel electrophoresis of restriction enzyme digests of bacterial DNA to confirm that the Proteus strains present in catheter biofilms were identical to the Proteus strains from the same patients' urine.

$11 \bullet$. Anderson GG, Palermo JJ, Schilling JD, et al. Intracellular bacterial biofilm-like pods in urinary tract infections. Science. 2003; 301:105-107. [PubMed: 12843396] This article documents the discovery of intracellular biofilm-like pods of Escherichia coli in mouse bladder epithelial cells. These pods of biofilm can explain the persistence of uropathogens in the bladder despite urinary antibiotics and host defenses. The accompanying photographs are impressive.

12. Nickel JC, Costerton JW, McLean RJC, Olson M. Bacterial biofilms: influence on the pathogenesis, diagnosis and treatment of urinary tract infections. J Antimicrobial Chemother. 1994; 33:31-41. 
13•. Beiko DT, Knudsen BE, Watterson JD, et al. Urinary tract biomaterials. J Urol. 2004; 171:24382444. [PubMed: 15126872] This article reviews the biomaterials currently used in the urinary tract and addresses issues of biocompatibility, encrustation, infection, and coatings.

14••. Brosnahan, J.; Jull, A.; Tracy, C. The Cochrane Library. Update Software; Oxford: 2004. Types of urethral catheters for managment of short-term voiding problems in hospitalised adults [Cochrane Review]. This is the definitive review of the effect of type of indwelling urethral catheter on the risk of urinary tract infection in adults who undergo short-term urinary catheterization. The types of catheters reviewed include antiseptic impregnated catheters (silver), antibiotic impregnated catheters (minocycline/rifampin), and silicone catheters.

15. Karchmer TB, Giannetta ET, Muto CA, et al. A randomized crossover study of silver-coated urinary catheters in hospitalized patients. Arch Intern Med. 2000; 160:3294-3298. [PubMed: 11088092]

16. Maki DG, Tambyah PA. Engineering out the risk of infection with urinary catheters. Emerg Infect Dis. 2001; 7:1-13. [PubMed: 11266288]

17. Silver S. Bacterial silver resistance: molecular biology and uses and misuses of silver compounds. FEMS Microbiol Rev. 2003; 27:341-353. [PubMed: 12829274] This article discusses bacterial resistance to silver compounds on a molecular level. The author also takes a critical look at appropriate and inappropriate medical uses of silver compounds.

18• Richards CL, Hoffman KC, Bernhard JM, et al. Development and characterization of an infection inhibiting urinary catheter. ASAIO J. 2003; 49:449-453. [PubMed: 12918589] The authors discuss characteristics of a urinary catheter that contains chlorhexidine digluconate.

19•. Al-Habdan I, Sadat-Ali M, Corea JR, et al. Assessment of nosocomial urinary tract infections in orthopaedic patients: a prospective and comparative study using two different catheters. Int Surg. 2003; 88:152-154. [PubMed: 14584770] This trial of an antibiotic-impregnated urinary catheter (nitrofuroxone) demonstrated the shortcomings typical of this genre: small sample size, lack of blinding, and unclear definition of outcomes.

20•. Vapnek JM, Maynard FM, Kim J. A prospective randomized trial of the LoFric hydrophilic coated catheter versus conventional plastic catheter for clean intermittent catheterization. J Urol. 2003; 169:994-998. [PubMed: 12576829] This article has a good discussion of the relationship between various catheter coating materials and urethral trauma.

21 . Balaban N, Gov Y, Giacometti A, et al. A chimeric peptide composed of a dermaseptin derivative and an RNA III-inhibiting peptide prevents graft-associated infections by antibiotic-resistant staphylococci. Antimicrob Agents Chemother. 2004; 48:2544-2550. [PubMed: 15215107] Although this article concerns vascular grafts, the strategy of using signaling peptides to disrupt biofilm formation is relevant to prevention of CAUTI.

22•. Balaban N, Giacometti A, Cirioni O, et al. Use of the quorum-sensing inhibitor RNAIII-inhibiting peptide to prevent biofilm formation in vivo by drug-resistant Staphylococcus epidermidis. J Infect Dis. 2003; 187:625-630. [PubMed: 12599079] This article describes one of the first trials to demonstrate that interfering with quorum-sensing can prevent biofilm formation by staphylococci.

23•. Dell'Acqua G, Giacometti A, Cirioni O, et al. Suppression of drug-resistant staphylococcal infections by the quorum-sensing inhibitor RNAIII-inhibiting peptide. J Infect Dis. 2004; 190:318-320. [PubMed: 15216467] In this rat model, application of a quorum-sensing inhibitor both locally and systemically reduced staphylococcal infection of vascular grafts.

24. Davies DG, Parsek MR, Pearson JP, et al. The involvement of cell-to-cell signals in the development of a bacterial biofilm. Science. 1998; 280:295-298. [PubMed: 9535661]

25. Ren D, Sims JJ, Wood TK. Inhibition of biofilm formation and swarming of Escherichia coli by (5Z)-4-bromo-5-(bromomethylene)-3-butyl-2(5H)-furanone. Environ Microbiol. 2001; 3:713-736.

26• Tenke P, Riedl CR, Jones GL, et al. Bacterial biofilm formation on urologic devices and heparin coating as a preventative strategy. Int J Antimicrob Agents. 2004; 23S1:S67-S74. [PubMed: 15037330] This review article incorporates previously unpublished clinical data concerning heparin-coated urologic stents.

27•. Marchese A, Bozzolasco M, Gualco L, et al. Effect of fosfomycin alone and in combination with $\mathrm{N}$-acetylcysteine on E. coli biofilms. Int J Antimicrob Agents. 2003; 22:S95-S100. In-vitro 
adding of $\mathrm{N}$-acetylcysteine to the growth media decreased biofilm formation by $E$. coli on polystyrene wells.

28. Alem MAS, Douglas LJ. Effects of aspirin and other nonsteroidal anti-inflam-matory drugs on biofilms and planktonic cells of Candida albicans. Antimicrob Agents Chemother. 2004; 48:4147. [PubMed: 14693516] This is the most recent article of several from the past 12 years, all of which suggest that non-steroidal antiinflammatory drugs decrease biofilm formation under laboratory conditions.

29•. Dromerick AW, Edwards DF. Relation of postvoid residual to urinary tract infection during stroke rehabilitation. Arch Phys Med Rehabil. 2003; 84:1369-1372. [PubMed: 13680576] This article confirms the accepted doctrine that urinary retention predisposes the patient to UTI.

30. Wilde MH, Carrigan MJ. A chart audit of factors related to urine flow and urinary tract infection. J Adv Nurs. 2003; 43:254-262. [PubMed: 12859784] This article likewise confirms that urinary obstruction predisposes the patient to UTI.

31 . Rosenthal VD, Guzman S, Safdar N. Effect of education and performance feedback on rates of catheter-associated urinary tract infection in intensive care units in Argentina. Infect Control Hosp Epidemiol. 2004; 25:47-50. [PubMed: 14756219] Implementation of education and performance feedback regarding catheter care was associated with decreased rates of CAUTI.

32. Misset B, Timset JF, Dumay MF, et al. A continuous quality-improvement program reduces nosocomial infection rates in the ICU. Intensive Care Med. 2004; 30:395-400. [PubMed: 14673521] Implementing Centers for Disease Control guidelines for prevention of UTI was associated with a decline in rates of CAUTI over 5 years.

33. Jain P, Parada JP, David A, Smith LG. Overuse of the indwelling urinary tract catheter in hospitalized medical patients. Arch Intern Med. 1995; 155:1425-1429. [PubMed: 7794092]

34. Munasinghe RL, Yazdani H, Siddique M, Hafeez W. Appropriateness of use of indwelling urinary catheters in patients admitted to the medical service. Infect Control Hosp Epidemiol. 2001; 22:647-649. [PubMed: 11776352]

35. Saint S, Wiese J, Amory JK, et al. Are physicians aware of which of their patients have indwelling urinary catheters? Am J Med. 2000; 109:476-480. [PubMed: 11042237]

36••. Cornia PB, Amory JK, Fraser S, et al. Computer-based order entry decreases duration of indwelling urinary catheterization in hospitalized patients. Am J Med. 2003; 114:404-407. [PubMed: 12714131] The authors test the hypothesis that putting an automatic stop date on urinary catheters will decrease days of unnecessary catheterization. Their positive finding invites a larger trial testing the impact of this intervention on CAUTI.

37••. Jepson, RG.; Mihaljevic, L.; Craig, J. The Cochrane Library. Update Software; Oxford: 2004. Cranberries for preventing urinary tract infections [Cochrane Review]. This is the definitive review of trials of cranberry products for prevention of UTI. The review is a good starting place for readers who would like to assess which trials are worth reading individually.

38. Kontiokari T, Sundqvist K, Nuutinen M, et al. Randomised trial of cranberry-lingonberry juice and Lactobacillus GG drink for the prevention of urinary tract infections in women. BMJ. 2001; 322:1571. [PubMed: 11431298]

39. Stothers L. A randomized trial to evaluate effectiveness and cost effectiveness of naturopathic cranberry products as prophylaxis against urinary tract infection in women. Can J Urol. 2002:1558-1562. 2002. [PubMed: 12121581]

40•. Raz R, Chazen B, Dan M. Cranberry juice and urinary tract infection. Clin Infect Dis. 2004; 38:1413-1419. [PubMed: 15156480] Like the Cochrane Review of the same topic, this is a good overview of studies investigating the use of cranberry juice to prevent UTI.

41. Darouiche RO, Donovan WH, Del Terzo M, et al. Pilot trial of bacterial interference for preventing urinary tract infection. Urology. 2001; 58:339-344. [PubMed: 11549475]

42. Trautner BW, Darouiche RO, Hull RA, et al. Pre-inoculation of urinary catheters with Escherichia coli 83972 inhibits catheter colonization by Enterococcus faecalis. J Urol. 2002; 167:375-379. [PubMed: 11743359]

43•. Trautner BW, Hull RA, Darouiche RO. Escherichia coli 83972 inhibits catheter adherence by a broad spectrum of uropathogens. Urology. 2003; 61:1059-1062. [PubMed: 12736047] A catheter 
coating of benign Escherchia coli impeded subsequent catheter colonization by yeasts, Gramnegative bacteria, and enterococci.

44. Kunin CM, McCormick RD. Prevention of catheter-associated urinary-tract infections by sterile closed drainage. N Engl J Med. 1966; 274:1154-1161. 